\title{
Weak solutions of equations of complex Monge-Ampère type
}

\author{
by SŁawomir KoŁodziej (Bielsko-Biała and Kraków)
}

\begin{abstract}
We prove some existence results for equations of complex Monge-Ampère type in strictly pseudoconvex domains and on Kähler manifolds.

0. Introduction. In this paper we extend the results on the existence of bounded (resp. continuous) solutions of the complex Monge-Ampère equation

$$
\left(d d^{c} u\right)^{n}=d \mu
$$

(with a given positive measure $d \mu$, a plurisubharmonic solution $u$, and the wedge product on the left defined as in [BT1]) to the case of more general equations of Monge-Ampère type

$$
\left(d d^{c} u\right)^{n}=F(u, \cdot) d \mu .
$$

We shall assume throughout that $F(t, z) \geq 0$ is nondecreasing, continuous in the first variable and measurable in the second one.

One can study the Dirichlet problem for the equation (0.1) in a strictly pseudoconvex domain in $\mathbb{C}^{n}$ imposing a boundary condition

$$
\lim _{z \rightarrow x} u(z)=\varphi(x) \quad \text { for } x \in \partial \Omega
$$

with given $\varphi \in C(\partial \Omega)$ (see [BT1], [Ce], [CKNS], [K1]-[K3]). By [K1] this problem has a unique bounded solution, provided a bounded subsolution exists. We shall prove (Theorem 1.1 below) that the same conclusion holds for (0.2) when $F$ is bounded. Thus we generalize the results of Bedford-Taylor [BT2] and Cegrell [Ce]. We refer to [CKNS] for the study of the classical solutions of the equation. Furthermore, let the measure $d \mu$ be represented as $f d \lambda$ with $d \lambda$ denoting the Lebesgue measure and $f \geq 0$ belonging to the Orlicz space
\end{abstract}

2000 Mathematics Subject Classification: Primary 32U15; 32W20.

Key words and phrases: plurisubharmonic function, complex Monge-Ampère operator. Partially supported by KBN Grant No. PO3A 00313. 


$$
L^{\chi}(\Omega)=\left\{g \in L^{1}(\Omega): g \geq 0, \int_{\Omega} \chi(g) d \lambda<\infty\right\} .
$$

If

$$
\chi(t)=|t|(\log (1+|t|))^{n} h(\log (1+|t|)),
$$

where $h: \mathbb{R}_{+} \rightarrow(1, \infty)$ is an increasing function satisfying

$$
\int_{1}^{\infty}\left(y h^{1 / n}(y)\right)^{-1} d y<\infty
$$

then the Dirichlet problem for (0.1) has a unique continuous solution (see [K2], [K3]). Corollary 1.2 says that this is still true for $(0.2)$ with $d \mu=d \lambda$ and $F(t, z) \leq f(z) \in L^{\chi}(\Omega)$.

In the next section we study the complex Monge-Ampère equation on a compact Kähler manifold $M$ with the fundamental form $\omega$ :

$$
\left(\omega+d d^{c} u\right)^{n}=F(u, \cdot) \omega^{n} .
$$

By the Stokes theorem, if $u$ satisfies $\left(0.2^{\prime}\right)$ then

$$
\int_{M} F(u, \cdot) \omega^{n}=\int_{M} \omega^{n} .
$$

For $F$ positive and smooth, satisfying the normalizing condition (2.1), the equation has been solved by Aubin [A1], [A2] and Yau [Y]. It is particularly interesting for $F(t, z)=\exp (\alpha t+f(z))$ when the solution serves to produce Kähler-Einstein metrics (see [A1]-[A3], [S], [Y]). Using a result from [K3], where the case of $F$ not depending on $t$ was treated, we show in Theorem 2.1 that for $F$ only nonnegative and $F \in L^{\chi}(M), \chi$ as above, one can find a continuous solution of $\left(0.2^{\prime}\right)$. This result, applied to $M=\mathbb{P}^{n}$, leads to solving (0.2) in the family of entire plurisubharmonic functions of minimal growth.

\section{Equations of Monge-Ampère type in a strictly pseudoconvex} domain

THEOREM 1.1. Let $\Omega$ be a strictly pseudoconvex domain and $\varphi \in C(\partial \Omega)$. Suppose there exists $v \in \operatorname{PSH} \cap L^{\infty}(\Omega)$ such that $\left(d d^{c} v\right)^{n}=d \mu$ and $\lim _{z \rightarrow x} v(z)=\varphi(x)$ for $x \in \partial \Omega$. Furthermore assume that $F: \mathbb{R} \times \Omega \rightarrow \mathbb{R}$ is a bounded nonnegative function which is nondecreasing and continuous in the first variable and $d \mu$-measurable in the second one. Then there exists a unique bounded plurisubharmonic solution of the Dirichlet problem

$$
\begin{aligned}
& u \in \mathrm{PSH} \cap L^{\infty}(\Omega), \\
& \left(d d^{c} u\right)^{n}=F(u, \cdot) d \mu, \\
& \lim _{z \rightarrow x} u(z)=\varphi(x) \quad \text { for } x \in \partial \Omega .
\end{aligned}
$$


Pr o of. Without loss of generality we assume $F \leq 1$. As in $[\mathrm{Ce}]$ we shall use Schauder's fixed point theorem. Consider $L^{2}(\Omega, d \lambda)$ equipped with the weak topology. Let $h$ be the maximal plurisubharmonic function in $\Omega$ with the boundary data equal to $\varphi$. Then the set

$$
\mathcal{A}=\{u \in \operatorname{PSH}(\Omega): v \leq u \leq h\}
$$

is convex and bounded (thereby compact) in $L^{2}(\Omega, d \lambda)$. We define the mapping $\mathcal{F}: \mathcal{A} \rightarrow \mathcal{A}$ taking for $\mathcal{F}(u)$ the solution of

$$
w \in \mathcal{A}, \quad\left(d d^{c} w\right)^{n}=F(u, \cdot) d \mu .
$$

This solution exists by [K1]. We need to show that $\mathcal{F}$ is continuous. Let $u_{j} \rightarrow u$ in $\mathcal{A}$. Set $w=\mathcal{F}(u)$ and $w_{j}=\mathcal{F}\left(u_{j}\right)$. By Hartogs' lemma, $u=$ $\left(\limsup u_{j}\right)^{*}$. Consider the auxiliary functions $\widetilde{u}_{k}=\left(\sup _{j \geq k} u_{j}\right)^{*}$ and $\widetilde{w}_{k}=\mathcal{F}\left(\widetilde{u}_{k}\right)$. Since $\left(d d^{c} \tilde{w}_{k}\right)^{n}$ is decreasing it follows from the comparison principle [BT3] that the sequence $\widetilde{w}_{k}$ is increasing to some $\widetilde{w} \in \mathcal{A}$. We also have

$$
\left(d d^{c} w_{k}\right)^{n}=F\left(u_{k}, \cdot\right) d \mu \leq F\left(\widetilde{u}_{k}, \cdot\right) d \mu=\left(d d^{c} \tilde{w}_{k}\right)^{n} .
$$

Hence, using the comparison principle, $\widetilde{w}_{k} \leq w_{k}$.

Furthermore, $\widetilde{u}_{k} \downarrow u$, and from the convergence theorem [BT3] one infers

$$
\left(d d^{c} \widetilde{w}\right)^{n}=\lim _{k \rightarrow \infty} F\left(\widetilde{u}_{k}, \cdot\right) d \mu=F(u, \cdot) d \mu .
$$

Since the solution to the Dirichlet problem (1.2) is unique we thus get

$$
w=\widetilde{w}=\lim \uparrow \widetilde{w}_{k} \leq \liminf w_{k} .
$$

It remains to prove that

$$
\limsup w_{k} \leq w .
$$

For this consider the sequence $\widehat{w}_{k}$ of functions in $\mathcal{A}$ solving

$$
\left(d d^{c} \widehat{w}_{k}\right)^{n}=F\left(\widehat{u}_{k}, \cdot\right) d \mu,
$$

where $\widehat{u}_{k}=\inf _{k<j} u_{j}$. Then, by the comparison principle, $w_{k} \leq \widehat{w}_{k}$ and $\widehat{w}_{k}$ decreases to $\widehat{w} \in A$. Since $\widehat{u}_{k} \uparrow u$ we obtain, applying the convergence theorem,

$$
\left(d d^{c} \widehat{w}\right)^{n}=\lim \uparrow\left(d d^{c} \widehat{w}_{k}\right)^{n}=F(u, \cdot) d \mu .
$$

This implies $w=\widehat{w}=\lim \downarrow \widehat{w}_{k} \geq \lim \sup w_{k}$. Thus we have proved the continuity of the mapping $\mathcal{F}$. The Schauder theorem now says that $\mathcal{F}$ has a fixed point, which gives the existence part of the statement. Uniqueness follows in a routine manner from the comparison principle and the fact that $F(\cdot, z)$ is nondecreasing. Suppose $u$ and $v$ solve our equation and $\{u<v+h\}$ is nonempty for some negative strictly plurisubharmonic function $h$. Then 
by the comparison principle [BT3],

$$
\begin{aligned}
& \int_{\{u<v+h\}}\left(d d^{c} v\right)^{n}+\left(d d^{c} h\right)^{n} \leq \int_{\{u<v+h\}}\left(d d^{c} u\right)^{n} \\
& =\int_{\{u<v+h\}} F(u, \cdot) d \mu \leq \int_{\{u<v+h\}} F(v, \cdot) d \mu=\int_{\{u<v+h\}}\left(d d^{c} v\right)^{n},
\end{aligned}
$$

which is impossible since the set over which we integrate has positive Lebesgue measure.

REMARK. The above theorem remains true for hyperconvex domains provided there exists a maximal plurisubharmonic function with boundary data equal to $\varphi$.

REMARK. The existence part of the theorem still holds when we drop the hypothesis that $F(\cdot, z)$ be nondecreasing, but uniqueness is then lost (see $[\mathrm{Ce}]$ ).

Corollary 1.2. Let $\Omega, \varphi$ and $F$ be as in the above theorem except that instead of assuming that $F$ is bounded we now suppose

$$
F(t, \cdot) \leq \psi \in L^{\chi}(\Omega),
$$

with $\chi$ given by (0.3). Then there exists a continuous solution to

$$
\begin{aligned}
& u \in \operatorname{PSH}(\Omega) \cap C(\bar{\Omega}), \\
& \left(d d^{c} u\right)^{n}=F(u, \cdot) d \lambda, \\
& \lim _{z \rightarrow x} u(z)=\varphi(x) \quad \text { for } x \in \partial \Omega,
\end{aligned}
$$

where $d \lambda$ denotes the Lebesgue measure.

$\operatorname{Pr}$ o o f. For the subsolution required in Theorem 1.1 we take $v \in \operatorname{PSH}(\Omega)$ $\cap C(\bar{\Omega})$ solving

$$
\left(d d^{c} v\right)^{n}=\psi d \lambda,
$$

with given boundary data. The existence of such a $v$ and continuity of $u$ has been proved in $[\mathrm{K} 2]$ and $[\mathrm{K} 3]$.

2. Equations of Monge-Ampère type on Kähler manifolds. Our next result is concerned with the equation $\left(0.2^{\prime}\right)$.

THEOREM 2.1. Let $(M, \omega)$ be a compact Kähler manifold with fundamental form $\omega$. Assume $F: \mathbb{R} \times M \rightarrow \mathbb{R}, 0 \leq F(t, z) \leq \psi(z) \in L^{\chi}(M)$ (with $\chi$ as in $(0.3))$, is a function such that $F(\cdot, z)$ is continuous and nondecreasing, $F(t, \cdot)$ is measurable and

$$
\lim _{t \rightarrow-\infty} \int_{M} F(t, z) \omega^{n} \leq \int_{M} \omega^{n} \leq \lim _{t \rightarrow \infty} \int_{M} F(t, z) \omega^{n} .
$$


Then the equation

$$
\mathcal{M} u:=\left(d d^{c} u+\omega\right)^{n}=F(u, \cdot) \omega^{n}
$$

has a continuous solution.

We shall need the following lemma.

Lemma 2.2. Let $(M, \omega)$ and $F$ be as in Theorem 2.1 except that we replace the condition (2.1) by a slightly stronger one:

$$
\lim _{t \rightarrow-\infty} \int_{M} F(t, z) \omega^{n}<\int_{M} \omega^{n}<\lim _{t \rightarrow \infty} \int_{M} F(t, z) \omega^{n} .
$$

Then there exists a sequence $F_{k} \in C^{\infty}(\mathbb{R} \times M)$ with the following properties: $F_{k}>0$,

$$
\frac{\partial}{\partial t} F_{k}(t, z) \geq 0, \quad \sup _{k} \int_{M} \chi\left(F_{k}\right) \omega^{n}<c<\infty,
$$

$\left(2.1^{\prime}\right)$ is fulfilled with $F_{k}$ in place of $F$, and for any bounded function $u$ in $M$,

$$
\lim _{k \rightarrow \infty} F_{k}(u(z), z)=F(u(z), z)
$$

almost everywhere in $M$.

Proof. Using $\left(2.1^{\prime}\right)$ we fix $R>0$ such that

$$
\int_{M} F(-R, z) \omega^{n}+R^{-1}<\int_{M} \omega^{n}<\int_{M} F(R, z) \omega^{n}-R^{-1} .
$$

For any positive integer $k>R$ we construct $F_{k}(t, z)$ as follows. First, note that the assumptions on $F(\cdot, z)$ allow us to find for given $z \in M$ a positive integer $N$ such that

$$
F\left(t_{j+1}, z\right)-F\left(t_{j}, z\right)<2^{-k-4}
$$

where

$$
t_{j}:=-k+j 2^{-N}, \quad j=0,1, \ldots, k 2^{N+1} .
$$

We fix $N$ such that (2.3) holds true for all $z \notin E$ with

$$
\int_{E} \omega^{n}<2^{-k-2} .
$$

In the next step we apply Luzin's theorem to choose $g_{j} \in C(M), g_{j} \geq 0$, $\int_{M} \chi\left(g_{j}\right) \omega^{n}<c$ satisfying

$$
\int_{E_{j}^{\prime}} \omega^{n}<2^{-k-j-3}, \quad E_{j}^{\prime}:=\left\{g_{j} \neq F\left(t_{j}, \cdot\right)\right\} .
$$

Set $h_{j}=\min _{s \geq j} g_{s}$. Then $\left\{h_{j} \neq F\left(t_{j}, \cdot\right)\right\} \subset E^{\prime}:=\bigcup_{s=0}^{N} E_{s}^{\prime}$. Since $h_{j} \leq$ $h_{j+1}$ one can approximate $h_{j}$ by smooth positive $f_{j}$ such that $f_{j} \leq f_{j+1}$, 
$\int_{M} \chi\left(f_{j}\right) \omega^{n}<c$ and

$$
\left\{\left|f_{j}-F\left(t_{j}, \cdot\right)\right|>2^{-k-3}\right\} \subset E^{\prime} .
$$

Fix $\psi_{j}$ smooth in a neighbourhood of the interval $\left[t_{j-1}, t_{j}\right]$ with $0 \leq \psi_{j}$ $\leq 1, \psi_{j}=1$ close to $t_{j}$ and $\psi_{j}=0$ close to $t_{j-1}$. Set

$$
F_{k}(t, z)=\psi_{j}(t) f_{j+1}(z)+\left(1-\psi_{j}(t)\right) f_{j}(z), \quad t \in\left[t_{j-1}, t_{j}\right] .
$$

By our choice of $\psi_{j}$ those functions are smooth and the choice of $f_{j}$ guarantees that $F_{k}$ is nondecreasing in $t$, satisfies the inequalities

$$
\int_{M} F_{k}(-R, z) \omega^{n}<\int_{M} \omega^{n}<\int_{M} F_{k}(R, z) \omega^{n},
$$

and the condition

$$
\sup _{k} \int_{M} \chi\left(F_{k}\right) \omega^{n}<c<\infty .
$$

Note that for $z \notin E^{\prime}$ and $t \in\left[t_{j-1}, t_{j}\right]$ we have (see (2.6))

$$
F_{k}(t, z) \geq f_{j}(z) \geq F\left(t_{j}, z\right)-2^{-k-3} \geq F(t, z)-2^{-k-3} .
$$

If moreover $z \notin E$ then (see (2.3) and (2.6))

$$
\begin{aligned}
F(t, z) & \geq F\left(t_{j-1}, z\right)>F\left(t_{j+1}, z\right)-2^{-k-3} \\
& \geq f_{j+1}(z)-2^{-k-2} \geq F_{k}(t, z)-2^{-k-2} .
\end{aligned}
$$

By (2.4) and (2.5) we have

$$
\int_{E \cup E^{\prime}} \omega^{n}<2^{-k-1}
$$

and writing $E=E(k)$ and $E^{\prime}=E^{\prime}(k)$ to indicate the dependence of those sets on $k$ we get

$$
\sum_{k=j}^{\infty} \int_{E(k) \cup E^{\prime}(k)} \omega^{n}<2^{-j} .
$$

Since, by (2.7) and (2.8), for any $z \notin \bigcup_{j \leq k}\left[E(k) \cup E^{\prime}(k)\right]$ and any $t$ we have $\lim _{k \rightarrow \infty} F_{k}(t, z)=F(t, z)$ the last part of the statement follows.

Proof of Theorem 2.1. First we prove the statement under the extra hypothesis $\left(2.1^{\prime}\right)$. We fix a sequence $F_{k}$ as in the above lemma. Yau's theorem [Y, Theorem 4] provides a smooth $u_{k}$ satisfying

$$
\mathcal{M} u_{k}=F_{k}\left(u_{k}, \cdot\right) \text {. }
$$

Let us define some auxiliary functions:

$$
u_{j k}=\max _{j \leq l \leq k} u_{l}, \quad v_{j}=\left(\lim _{k \rightarrow \infty} u_{j k}\right)^{*}, \quad u=\left(\lim \sup u_{k}\right)^{*} .
$$


One can apply [K3, Section 2.3] to conclude that

$$
\sup _{M} u_{k}-\inf _{M} u_{k}<c_{0}
$$

with $c_{0}$ depending only on $c$ from the assumptions. By Stokes' theorem,

$$
\int_{M} F\left(u_{k}, \cdot\right) \omega^{n}=\int_{M} \omega^{n}
$$

So, in view of $\left(2.2^{\prime}\right)$ we obtain

$$
\sup _{M} u_{k}<R, \quad \inf _{M} u_{k}>-R, \quad k>R .
$$

Those inequalities and (2.9) imply that the sequence $u_{k}$ is uniformly bounded. Passing to a subsequence we assume $u_{k} \rightarrow u$ a.e. Applying [BT1, Proposition 2.8] one gets

$$
\mathcal{M} u_{j k} \geq \min _{j \leq l \leq k} F_{l}\left(u_{l}, \cdot\right)
$$

Now, for fixed $\varepsilon>0$ we find an integer $j_{0}$ and a set $E$ with

$$
u_{j}(z) \geq u(z)-\varepsilon, \quad j \geq j_{0}, z \notin E,
$$

and $\int_{E} \omega^{n}<\varepsilon$. Then for $j, z$ as above,

$$
\mathcal{M} u_{j k}(z) \geq \inf _{j \leq l \leq k} F_{l}(u(z)-\varepsilon, z) .
$$

Letting $k$ to $\infty$ and using the convergence theorem [BT3] we obtain

$$
\mathcal{M} v_{j}(z) \geq \inf _{j \leq l} F_{l}(u(z)-\varepsilon, z), \quad z \notin E .
$$

Since $v_{j}$ decreases to $u$ one can apply the convergence theorem and Lemma 2.2 to get

$$
\mathcal{M} u(z) \geq F(u(z)-\varepsilon, z) \quad \text { a.e. in } M \backslash E .
$$

This is true for any $\varepsilon>0$ and $F(\cdot, z)$ is continuous, so

$$
\mathcal{M} u(z) \geq F(u(z), z) \quad \text { a.e. }
$$

Since by the lemma and the argument above $F_{k}\left(u_{k}(z), z\right) \rightarrow F(u(z), z)$ almost everywhere and, on the other hand,

$$
\int_{M} F_{k}\left(u_{k}, \cdot\right) \omega^{n}=\int_{M} \omega^{n}
$$

for any $k$, we conclude that the integrals over $M$ of both sides of inequality (2.10) are equal. So the functions are equal. To get the general case, note that if we had equalities in $\left(2.1^{\prime}\right)$ then $F$ would be independent of $t$ and the equation would reduce to the one solved in [K3]. If we have one strict inequality we can find a monotone sequence $c_{j} \rightarrow 0$ such that for any $j$,

$$
\lim _{t \rightarrow-\infty} \int_{M}\left(F(t, z)+c_{j}\right) \omega^{n}<\int_{M} \omega^{n}<\lim _{t \rightarrow \infty} \int_{M}\left(F(t, z)+c_{j}\right) \omega^{n} .
$$


Then by the preceding part of the proof, there exist $u_{j}$ satisfying

$$
\mathcal{M} u_{j}=F\left(u_{j}(z), z\right)+c_{j} .
$$

The function $u=\left(\lim \sup u_{k}\right)^{*}$ is the desired solution as can be seen by repeating the above reasoning.

Theorem 2.1, when applied to $M=\mathbb{P}^{n}$ equipped with the Fubini-Study metric, allows us to solve the Monge-Ampère type equation in the class of entire plurisubharmonic functions of minimal growth, usually denoted by $\mathcal{L}_{+}$:

$$
\mathcal{L}_{+}=\left\{u \in \operatorname{PSH}\left(\mathbb{C}^{n}\right):\left|u(z)-\frac{1}{2} \log \left(1+|z|^{2}\right)\right|<\text { const }\right\} .
$$

The function

$$
v_{0}(z)=\frac{1}{2} \log \left(1+|z|^{2}\right) \in \mathcal{L}_{+}
$$

is a potential of the Fubini-Study metric in $\mathbb{P}^{n}$ restricted to $\mathbb{C}^{n}$ (which is embedded in the standard way). We have

$$
\left(d d^{c} v_{0}\right)^{n}=\omega^{n}=\frac{n !}{\left(1+|z|^{2}\right)^{n+1}} d \lambda=(2 \pi)^{n} .
$$

Let $F: \mathbb{R} \times \mathbb{C}^{n} \rightarrow \mathbb{R}, 0 \leq F(t, z)$, be continuous and nondecreasing in $t$, measurable in $z$ and such that for some $t_{0}$,

$$
\int_{\mathbb{C}^{n}} F\left(t_{0}, z\right) d \lambda=(2 \pi)^{n} .
$$

Suppose also that

$$
F(t, z) \leq f(z)\left(1+|z|^{2}\right)^{-n-1}
$$

with $f \in L^{\chi}\left(\omega^{n}\right)$.

Corollary 2.3. For F introduced above the equation

$$
u \in \mathcal{L}_{+}, \quad\left(d d^{c} u\right)^{n}=F\left(u-v_{0}, \cdot\right) d \lambda
$$

has a solution.

The uniqueness of those solutions has been shown in [BT4] in the case when $F$ is independent either of $t$ or $z$.

\section{References}

[A1] T. Aubin, Equations du type Monge-Ampère sur les variétés kählériennes compactes, C. R. Acad. Sci. Paris 283 (1976), 119-121.

[A2] - Equations du type Monge-Ampère sur les variétés kählériennes compactes, Bull. Sci. Math. 102 (1978), 63-95.

[A3] —, Nonlinear Analysis on Manifolds. Monge-Ampère Equations, Grundlehren Math. Wiss. 244, Springer, 1982. 
[BT1] E. Bedford and B. A. Taylor, The Dirichlet problem for the complex MongeAmpère operator, Invent. Math. 37 (1976), 1-44.

[BT2] - - - The Dirichlet problem for an equation of complex Monge-Ampère type, in: Partial Differential Equations and Geometry, C. Byrnes (ed.), Dekker, 1979, $39-50$.

[BT3] E. Bedford and B. A. Taylor, A new capacity for plurisubharmonic functions, Acta Math. 149 (1982), 1-40.

[BT4] -, 一, Uniqueness for the complex Monge-Ampère equation for functions of logarithmic growth, Indiana Univ. Math. J. 38 (1989), 455-469.

[CKNS] L. Caffarelli, J. J. Kohn, L. Nirenberg and J. Spruck, The Dirichlet problem for nonlinear second-order elliptic equations. II. Complex Monge-Ampère, and uniformly elliptic, equations, Comm. Pure Appl. Math. 38 (1985), 209-252.

[Ce] U. Cegrell, On the Dirichlet problem for the complex Monge-Ampère operator, Math. Z. 185 (1984), 247-251.

[K1] S. Kołodziej, The range of the complex Monge-Ampère operator II, Indiana Univ. Math. J. 44 (1995), 765-782.

[K2] - Some sufficient conditions for solvability of the Dirichlet problem for the complex Monge-Ampère operator, Ann. Polon. Math. 65 (1996), 11-21.

[K3] -, The complex Monge-Ampère equation, Acta Math. 180 (1998), 69-117.

[S] Y.-T. Siu, Lectures on Hermitian-Einstein Metrics for Stable Bundles and Kähler-Einstein Metrics, Birkhäuser, 1987.

[Y] S.-T. Yau, On the Ricci curvature of a compact Kähler manifold and the complex Monge-Ampère equation, Comm. Pure Appl. Math. 31 (1978), 339-411.

Technical University of Łódź

Branch in Bielsko-Biała

Willowa 2

43-300 Bielsko-Biała, Poland
Institute of Mathematics Jagiellonian University Reymonta 4

30-059 Kraków, Poland

E-mail: kolodzie@im.uj.edu.pl

Reçu par la Rédaction le 19.1.1999

Révisé le 29.11.1999 